\title{
COMPARISON OF FENTANYL AND BUTORPHANOL IN ATTENUATING THE HAEMODYNAMIC RESPONSES TO LARYNGOSCOPY AND ENDOTRACHEAL INTUBATION
}

\author{
Solaiappan Balasubramaniam ${ }^{1}$, Revathy Jeevarathnam ${ }^{2}$ \\ ${ }^{1}$ Associate Professor, Department of Anaesthesiology, Chengalpattu Medical College, Chengalpattu. \\ 2 Professor, Department of Anaesthesiology, Chengalpattu Medical College, Chengalpattu.
}

\section{ABSTRACT}

\section{BACKGROUND}

Numerous attempts have been made in the past to attenuate the pressor response that arises from the manipulation of the airway, attributed to the sympathoadrenal discharge because of stimulation of epipharyngeal and parapharyngeal regions. This study compares the effects of two synthetic opioids Fentanyl and Butorphanol in attenuating the pressor response to laryngoscopy and intubation.

The aim of this study is to compare the pressor response to laryngoscopy and intubation using intravenous Fentanyl and Butorphanol on the following parameters: (1) Heart Rate, (2) Systolic BP, (3) Diastolic BP, (4) Mean Arterial Pressure and (5) Rate Pressure Product.

\section{MATERIALS AND METHODS}

This study is a double-blind randomised trial comparing two opioid drugs, Fentanyl and Butorphanol; it was carried out at the Department of Anaesthesiology, Chengalpattu Medical College, Tamilnadu. With the Institutional Ethical Committee approval, 60 ASA PS I patients of either sex of age between 20 and 50 years scheduled for elective surgeries under general anaesthesia were enrolled into two groups, Group B - Inj. Butorphanol $40 \mu \mathrm{g} / \mathrm{kg}$ IV and Group F - Inj. Fentanyl $2 \mu \mathrm{g} / \mathrm{kg}$ IV administered 5 minutes before Laryngoscopy and Intubation. Haemodynamic parameters were recorded for up to 10 minutes after endotracheal intubation.

Statistical Analysis - The results were systematically analysed using paired " $\mathrm{t}$ " test for variables within the group and unpaired " $\mathrm{t}$ " test for variables between the two study groups. Software used for analysis of data was SPSS for windows. P value of less than 0.05 was considered statistically significant.

\section{RESULTS}

We observed that the rise in haemodynamic parameters following laryngoscopy and intubation was significantly lower in the Butorphanol group when compared to the Fentanyl group. The parameters like HR, Diastolic BP, mean arterial pressure and rate pressure product were short-lived in the Butorphanol group.

\section{CONCLUSION}

Butorphanol has a significant advantage over an equipotent dose of fentanyl in the sense that the increase in the stress response was comparatively less and of shorter duration.

\section{KEYWORDS}

Fentanyl, Butorphanol, Laryngoscopy, Intubation.

HOW TO CITE THIS ARTICLE: Balasubramaniam S, Revathy J. Comparison of fentanyl and butorphanol in attenuating the haemodynamic responses to laryngoscopy and endotracheal intubation. J. Evolution Med. Dent. Sci. 2016;5(99):7288-7293, D0I: $10.14260 /$ jemds/2016/1649

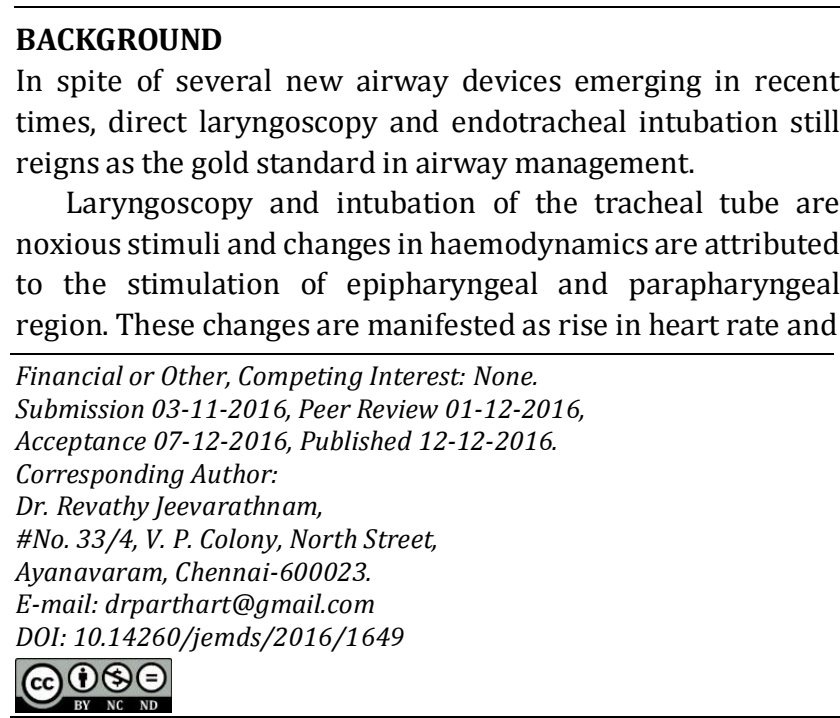
blood pressure which are variable, transient and unpredictable.

Although, a normotensive patient can tolerate this response, it may pose serious challenges in hypertension, coronary artery disease that intracranial compliance like extra- or intra-dural haematoma tumours or aneurysms, left ventricular failure, myocardial ischaemia and cerebral haemorrhage can be precipitated by sudden changes in haemodynamics.

Some pharmacological agents used to suppress the pressor response are volatile anaesthetic agents ${ }^{1}$ - lignocaine, ${ }^{2}$ opioids, ${ }^{3}$ vasodilators (Sodium nitroprusside, ${ }^{4}$ nitroglycerine, ${ }^{5}$ calcium channel blockers 6 and adrenergic blockers ${ }^{7}$ ). The nonpharmacological methods include smooth and gentle intubation with a shorter duration of laryngoscopy, use of $\mathrm{LMA}^{8}$ and blocking glossopharyngeal and superior laryngeal nerves. ${ }^{9}$ 
Opioids have been employed for this purpose for quite some time and has been proved extremely useful in attenuating the pressor response apart from providing good analgesia.

Hence, in this study we have decided to compare the effects of two opioids, fentanyl and Butorphanol, in attenuating the pressor response to laryngoscopy and intubation.

Fentanyl 10 is a phenyl piperidine derivative synthetic opioid agonist that is structurally related to meperidine. As an analgesic, fentanyl is 75 to 125 times more potent than morphine. A single dose of fentanyl administered intravenously has a more rapid onset and shorter duration of action than morphine. Low doses of fentanyl, 1 to $2 \mu \mathrm{g} / \mathrm{kg} \mathrm{IV}$, are injected to provide analgesia. Fentanyl, 2 to $20 \mu \mathrm{g} / \mathrm{kg} \mathrm{IV}$, may be administered as an adjuvant to inhaled anaesthetics in an attempt to blunt circulatory responses to direct laryngoscopy for intubation of the trachea. A unique aspect 11 of fentanyl's clinical application is that it can be delivered in numerous ways. In addition to the intravenous route, transdermal, transmucosal, transnasal and transpulmonary routes can also deliver fentanyl.

Butorphanol is an agonist-antagonist opioid, an agonist at $\mathrm{K}$-receptors. Its activity at $\mu$-receptors is either antagonistic or partially agonistic. It is five to eight times as potent as morphine and is available only in parenteral form, whereas the duration of action of butorphanol is similar to that of morphine, its plasma half-life is only 2 to 3 hours. Side effects after butorphanol include drowsiness, sweating, nausea and CNS stimulation.

\section{Aims and Objectives}

To compare the attenuation of pressure response to laryngoscopy and intubation with fentanyl and butorphanol using the following parameters.

1. Systolic BP.

2. Diastolic BP.

3. Mean arterial pressure.

4. Mean pulse rate.

5. Rate pressure product.

\section{MATERIALS AND METHODS}

This is a double-blind randomised trial study comparing the two opioids, fentanyl and butorphanol, was carried out in Govt. Chengalpattu Medical College Hospital, Tamilnadu, after obtaining the Institutional Ethical Committee approval and written informed consent from the patients.

Sixty patients were randomly subjected to this study. Thirty patients were enrolled into Group F and thirty patients were enrolled into Group B.

\section{Inclusion Criteria}

1. ASA PS I.

2. 20 - 50 years of age.

3. Both sex.

4. Elective procedure under GA.

5. Mallampati airway class I and II.

\section{Exclusion Criteria}

1. Hypertension.

2. Valvular heart disease.

3. Ischaemic heart disease.

4. Endocrine disorders.
5. Metabolic disorders.

6. Respiratory disease.

7. Anaemia.

8. Allergic diathesis.

9. Unanticipated difficult airway.

The two drugs fentanyl and butorphanol were compared in their efficiency in attenuating the pressor response to laryngoscopy and endotracheal intubation in terms of their effect on heart rate, systolic $\mathrm{BP}$, diastolic $\mathrm{BP}$, mean arterial pressure, rate pressure product.

All patients were examined preoperatively and assessed with history, physical examination and necessary laboratory investigations. Patients were randomly divided into two groups: Group B (Butorphanol) and Group F (Fentanyl).

On the day of surgery, all patients were reassessed in the patient's waiting room. Heart rate, systolic BP, diastolic BP and mean arterial pressure were recorded and noted as the first preoperative value (Pre-Op 1). All patients received Inj. Glycopyrrolate $0.005 \mathrm{mg} / \mathrm{kg}$ and Inj. Midazolam $0.05 \mathrm{mg} / \mathrm{kg}$ intramuscularly as premedication. Thirty minutes later the patients were shifted inside the operating room. Heart rate, systolic BP, diastolic BP, mean arterial pressure were recorded and noted as second preoperative value (Pre-Op 2). This was taken as the baseline value for comparison of different parameters at various time intervals. After recording the baseline parameters, patients in Group F received Inj. Fentanyl $2 \mu \mathrm{g} / \mathrm{kg}$ and Group B received Inj. Butorphanol $40 \mu \mathrm{g} / \mathrm{kg}$ by intravenous route. Heart rate, systolic BP, diastolic BP and mean arterial pressure were recorded for 5 minutes and the fifth minute values were noted as the Pre-Induction values (PI). Occurrence of any haemodynamic variability was noted down, i.e. greater than $20 \%$ decrease in mean arterial pressure was defined as hypotension and less than 60 beats per minute was defined as bradycardia when compared to preoperative values (Pre-Op 2).

Five minutes after the administration of the test drug, patients were pre-oxygenated with $100 \%$ oxygen and induced with Inj. Thiopentone sodium $5 \mathrm{mg} / \mathrm{kg}$ IV as $2.5 \%$ solution. Inj. Vecuronium $0.1 \mathrm{mg} / \mathrm{kg}$ was given to achieve muscle relaxation and controlled positive pressure ventilation was done with $100 \%$ oxygen by mask. After 3 minutes, endotracheal intubation was done with appropriate sized endotracheal tube using the laryngoscope with Macintosh blade. Patients who strained more than 15 seconds of laryngoscopy and patients requiring a second attempt of laryngoscopy were excluded from the study.

Heart rate, BP (systolic, diastolic, mean arterial pressure) were recorded at the first, second, third, fourth, fifth and tenth minute following intubation and was noted as T1, T2, T3, T4, $\mathrm{T} 5$ and T10 respectively. No surgical stimulation was allowed for the first 10 minutes after intubation.

\section{Statistical Analysis}

The results were systematically analysed using paired " $\mathrm{t}$ " test for variables within the group and unpaired " $\mathrm{t}$ " test for variables between the two study groups. Software used for analysis of data was SPSS for windows. P value of less than 0.05 was considered statistically significant.

\section{RESULTS}

Sixty patients were subjected to this study. Thirty patients were enrolled into Group F and thirty patients were enrolled 
into Group B. There was no statistical difference between the two groups in respect to age, sex, weight and Mallampati Classification (MPC). The comparison of preoperative parameters like pulse rate, systolic BP, diastolic BP, mean arterial pressure showed no statistical difference between the two groups.
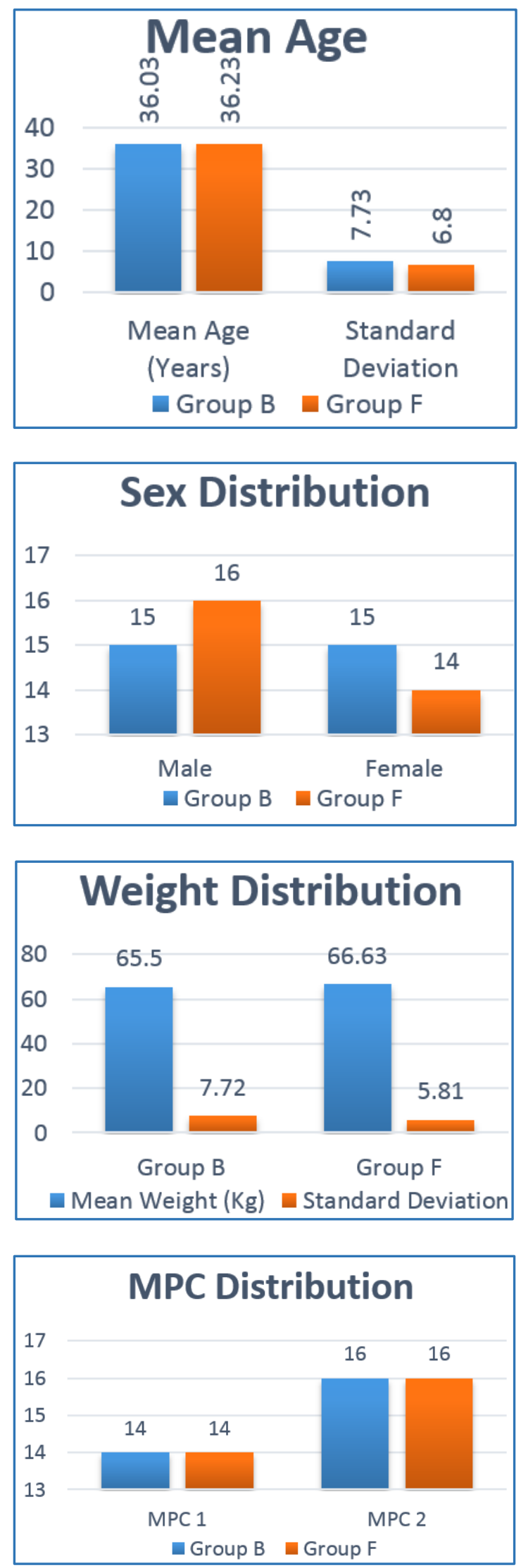

Comparison of the preoperative parameters before the premedication (Pre-Op 1) and the parameters after the premedication (Pre-Op 2) showed that there was no statistically significant difference between the two groups.

\begin{tabular}{|c|c|c|c|}
\hline Parameter & Group B & Group F & P value \\
\hline Pre-0p 1 HR & $81.4 \pm 4.65$ & $80.4 \pm 4.59$ & 0.2031 \\
\hline Pre-0p 1 SBP & $122.53 \pm 5.72$ & $123.53 \pm 4.83$ & 0.2339 \\
\hline Pre-op1 DBP & $81.33 \pm 5.47$ & $81.4 \pm 3.48$ & 0.4776 \\
\hline Pre-op1 MAP & $95.06 \pm 5.02$ & $95.44 \pm 2.35$ & 0.3553 \\
\hline \multicolumn{4}{|c|}{ Table 1. Comparison of } \\
Preoperative Parameters (Pre-Op 1)
\end{tabular}

\begin{tabular}{|c|c|c|c|}
\hline Parameter & Group B & Group F & P value \\
\hline Pre-Op 2 HR & $80.0 \pm 5.14$ & $77.67 \pm 4.95$ & 0.2846 \\
\hline Pre-Op 2 SBP & $120.96 \pm 6.36$ & $122.03 \pm 4.99$ & 0.2365 \\
\hline Pre-Op 2 DBP & $79.83 \pm 5.83$ & $80.73 \pm 3.44$ & 0.235 \\
\hline Pre-Op 2 MAP & $92.94 \pm 5.52$ & $94.5 \pm 2.40$ & 0.082 \\
\hline \multicolumn{4}{|c|}{ Table 2. Comparison of Preoperative } \\
Parameters (Pre-Op 2) \\
\hline
\end{tabular}

The mean heart rate is significantly lower in Group B when compared to Group F from the second and up to the tenth minute after intubation. The heart rate in Group B after intubation becomes comparable to the preoperative (Pre-Op 2) heart rate at the third minute after intubation and it becomes significantly lower than the preoperative heart rate from the fourth minute after intubation onwards. The heart rate in Group F after intubation becomes comparable to the preoperative (Pre-Op 2) heart rate at the fifth minute after intubation and it becomes significantly lower than the preoperative heart rate at the tenth minute after intubation onwards.

\begin{tabular}{|c|c|c|c|}
\hline $\begin{array}{l}\text { Mean Heart } \\
\text { Rate }\end{array}$ & Group B & Group F & $P$ value \\
\hline Pre-Op 1 & $81.4 \pm 4.65$ & $80.4 \pm 4.59$ & 0.2031 \\
\hline Pre-Op 2 & $80.0 \pm 5.14$ & $77.67 \pm 4.95$ & 0.2846 \\
\hline PI (Pre-Induction) & $77.13 \pm 5.30$ & $74.8 \pm 11.63$ & 0.1609 \\
\hline $\mathrm{T} 1$ & $88.93 \pm 4.63$ & $89.8 \pm 3.40$ & 0.2062 \\
\hline $\mathrm{T} 2$ & $84.73 \pm 4.97$ & $87.87 \pm 3.03$ & 0.0023 \\
\hline T3 & $80.87 \pm 5.17$ & $84.7 \pm 3.09$ & $<0.001$ \\
\hline $\mathrm{T} 4$ & $77.0 \pm 5.38$ & $81.33 \pm 2.94$ & $<0.001$ \\
\hline T5 & $73.07 \pm 4.40$ & $77.63 \pm 2.55$ & $<0.001$ \\
\hline T10 & $68.17 \pm 2.72$ & $71.9 \pm 2.39$ & $<0.001$ \\
\hline \multicolumn{4}{|c|}{ Table 3. Mean Heart Rate } \\
\hline
\end{tabular}

Mean systolic blood pressure is significantly lower in Group B when compared to Group F at the second and third minute after intubation. The systolic BP in Group B after intubation becomes comparable to the preoperative systolic $\mathrm{BP}$ at the fourth minute after intubation and it becomes significantly lower than the preoperative systolic BP at the tenth minute after intubation. The systolic BP in Group F after intubation becomes comparable to the preoperative systolic $\mathrm{BP}$ at the fourth minute after intubation and it becomes significantly lower than the preoperative systolic BP at the fifth minute after intubation. 


\begin{tabular}{|c|c|c|c|}
\hline Mean Systolic BP & Group B & Group F & P value \\
\hline Pre-Op 1 & $122.53 \pm 5.72$ & $122.53 \pm 4.83$ & 0.2340 \\
\hline Pre-Op 2 & $120.96 \pm 6.36$ & $122.03 \pm 4.99$ & 0.2365 \\
\hline PI (Pre-Induction) & $118.1 \pm 6.18$ & $119.13 \pm 15.31$ & 0.2452 \\
\hline T1 & $128.73 \pm 4.90$ & $130.33 \pm 2.55$ & 0.0592 \\
\hline T2 & $125.6 \pm 5.51$ & $128.33 \pm 2.41$ & 0.0079 \\
\hline T3 & $122.36 \pm 6.15$ & $124.46 \pm 2.75$ & 0.0467 \\
\hline T4 & $119.7 \pm 6.24$ & $121.26 \pm 2.80$ & 0.1076 \\
\hline T5 & $116.86 \pm 5.92$ & $117.73 \pm 3.07$ & 0.2399 \\
\hline T10 & $112.17 \pm 6.01$ & $113.06 \pm 2.92$ & 0.2341 \\
\hline \multicolumn{4}{|r}{ Table 4. Mean Systolic Blood Pressure } \\
\hline \multicolumn{4}{|l}{} \\
\hline \multicolumn{4}{|l}{}
\end{tabular}

Mean diastolic blood pressure is significantly lower in Group B when compared to Group F from immediately before induction and until the tenth minute after induction. The diastolic BP in Group B after intubation becomes comparable to the preoperative diastolic BP at the third minute after intubation and it becomes significantly lower than the preoperative diastolic BP at the tenth minute after intubation. The diastolic BP in Group F after intubation becomes comparable to the preoperative diastolic BP at the fourth minute after intubation and it becomes significantly lower than the preoperative diastolic BP at the tenth minute after intubation.

\begin{tabular}{|c|c|c|c|}
\hline Mean Diastolic BP & Group B & Group F & P value \\
\hline Pre-Op 1 & $81.33 \pm 5.47$ & $81.4 \pm 3.48$ & 0.4776 \\
\hline Pre-Op 2 & $79.83 \pm 5.83$ & $80.73 \pm 3.44$ & 0.2350 \\
\hline PI (Pre-Induction) & $77.3 \pm 5.01$ & $79.53 \pm 2.93$ & 0.0197 \\
\hline $\mathrm{T} 1$ & $86.23 \pm 4.56$ & $89.8 \pm 1.82$ & $<0.001$ \\
\hline $\mathrm{T} 2$ & $82.2 \pm 3.88$ & $88.26 \pm 1.85$ & $<0.001$ \\
\hline T3 & $78.93 \pm 4.31$ & $86.06 \pm 1.46$ & $<0.001$ \\
\hline T4 & $75.36 \pm 4.01$ & $84.67 \pm 1.24$ & $<0.001$ \\
\hline T5 & $71.9 \pm 3.82$ & $81.7 \pm 1.49$ & $<0.001$ \\
\hline T10 & $68.68 \pm 2.49$ & $77.3 \pm 2.74$ & 0.0033 \\
\hline
\end{tabular}

Mean arterial pressure is significantly lower in Group B when compared to Group F from immediately before induction and until the tenth minute after induction. The mean arterial pressure in Group B after intubation becomes comparable to the preoperative mean arterial pressure at the third minute after intubation and it becomes significantly lower than the preoperative mean arterial pressure from the fourth minute after intubation. The mean arterial pressure in Group F after intubation becomes comparable to the preoperative mean arterial pressure at the fourth minute after intubation and it becomes significantly lower than the preoperative mean arterial pressure at the tenth minute after intubation.

\begin{tabular}{|c|c|c|c|}
\hline $\begin{array}{l}\text { Mean Arterial } \\
\text { Pressure }\end{array}$ & Group B & Group F & $P$ value \\
\hline Pre-Op 1 & $95.07 \pm 5.02$ & $95.44 \pm 2.35$ & 0.3555 \\
\hline Pre-Op 2 & $92.94 \pm 5.52$ & $94.5 \pm 2.40$ & 0.082 \\
\hline PI (Pre-Induction) & $90.9 \pm 4.83$ & $92.73 \pm 2.45$ & 0.0346 \\
\hline $\mathrm{T} 1$ & $100.4 \pm 4.19$ & $103.31 \pm 1.52$ & $<0.001$ \\
\hline $\mathrm{T} 2$ & $96.67 \pm 4.07$ & $101.62 \pm 1.68$ & $<0.001$ \\
\hline T3 & $93.41 \pm 4.60$ & $98.86 \pm 1.71$ & $<0.001$ \\
\hline $\mathrm{T} 4$ & $90.14 \pm 4.27$ & $96.82 \pm 1.53$ & $<0.001$ \\
\hline T5 & $86.89 \pm 4.02$ & $93.71 \pm 1.54$ & $<0.001$ \\
\hline T10 & $80.41 \pm 15.54$ & $89.22 \pm 1.90$ & 0.002 \\
\hline
\end{tabular}

The Rate Pressure Product is significantly lower in Group $\mathrm{B}$ when compared to Group F from the second minute after intubation. The Rate Pressure Product in Group B after intubation becomes comparable to the preoperative Rate Pressure Product at the fourth minute after intubation and it becomes significantly lower than the preoperative Rate Pressure Product from the fifth minute after intubation. The rate pressure product in Group F after intubation becomes comparable to the preoperative Rate Pressure Product at the fifth minute after intubation and it becomes significantly lower than the preoperative Rate Pressure Product at the tenth minute after intubation.

\begin{tabular}{|c|c|c|c|}
\hline $\begin{array}{c}\text { Rate } \\
\text { Pressure } \\
\text { Product }\end{array}$ & Group B & Group F & $\begin{array}{c}\text { P } \\
\text { value }\end{array}$ \\
\hline Pre-0p 1 & $9988.03 \pm 913.21$ & $9943.67 \pm 855.9$ & 0.4003 \\
\hline Pre-0p 2 & $9550.96 \pm 1002.83$ & $9489.9 \pm 855.9$ & 0.4003 \\
\hline $\begin{array}{c}\text { PI (Pre- } \\
\text { Induction) }\end{array}$ & $9124.00 \pm 945.37$ & $8909.7 \pm 1487.18$ & 0.254 \\
\hline T1 & $11459.1 \pm 888.94$ & $11706.83 \pm 564.61$ & 0.101 \\
\hline T2 & $10657.6 \pm 962.02$ & $11278.43 \pm 496.34$ & 0.001 \\
\hline T3 & $9915.96 \pm 1023.89$ & $10546.27 \pm 533.07$ & 0.002 \\
\hline T4 & $9237.56 \pm 1008.6$ & $9864.73 \pm 461.28$ & 0.002 \\
\hline T5 & $8553.96 \pm 836.23$ & $9140.26 \pm 389.94$ & $<0.001$ \\
\hline T10 & $7400.67 \pm 1525.76$ & $8127.63 \pm 289.26$ & 0.0065 \\
\hline \multicolumn{4}{|l|}{ Table 7. Rate Pressure Product } \\
\hline
\end{tabular}

The complications noted in a few patients of both groups were hypotension, bradycardia, shivering, nausea and vomiting. The incidence of shivering was significantly lesser in butorphanol group than the fentanyl group.

\begin{tabular}{|c|c|c|c|c|}
\hline \multicolumn{2}{|c|}{ Complications } & Group B & Group F & P value \\
\hline \multirow{2}{*}{ Intraoperative } & Hypotension & 3 & 2 & 0.1869 \\
\cline { 2 - 5 } & Bradycardia & 3 & 1 & 0.2529 \\
\hline \multirow{3}{*}{ Postoperative } & Shivering & 2 & 6 & 0.0051 \\
\cline { 2 - 5 } & $\begin{array}{c}\text { Nausea and } \\
\text { Vomiting }\end{array}$ & 5 & 6 & 0.1704 \\
\hline \multicolumn{5}{|c|}{ Table 8. Complications } \\
\hline
\end{tabular}

\section{DISCUSSION}

Even in well-anaesthetised individuals, reflex tachycardia and hypertension on laryngoscopy and intubation occurs quite frequently.12 Many different opioid combinations and dosages have been tried in the past on obtunding this pressor response. In our study, we have compared IV Fentanyl and IV Butorphanol with 30 patients of each group.

King et al (1951) ${ }^{13}$ observed a transient but marked increase in systolic and diastolic BP during laryngoscopy. Similarly, in our study also a short duration of increase of these parameters were noted but significantly elevated BP when compared to the baseline values. We also observed a significant increase in mean arterial pressure with laryngoscopy compared to the preoperative values in both the study groups. Corbett and Kerr (1969) ${ }^{14}$ and Forbes and Dalhy (1970) ${ }^{15}$ had also observed similar changes that regressed gradually.

When heart rate following intubation was recorded at various time intervals between fentanyl and butorphanol group, the increase in heart rate in butorphanol group was 
significantly lower than the fentanyl group. This was similar to the observations made by Philip BK et al ${ }^{16}$ in their study.

We recorded an increase in both systolic and diastolic BP in both groups following intubation. The increase in systolic BP was significantly lower in Group B when compared to Group F, but only for short duration during the second and third minute after intubation. During the rest of the study period, it was comparable between the two groups. In contrast Group B showed a significant lowering of the diastolic BP from the time prior to induction to the end if the study period of ten minutes. Nevertheless, an increase was observed in both the groups following intubation. Philip BK et al and Vikramjeet Arora et $\mathrm{al}^{17}$ also observed this in their respective studies.

Yushi et al ${ }^{18}$ in his study concluded that $2 \mu \mathrm{g} / \mathrm{kg}$ fentanyl suppresses the haemodynamic response to endotracheal intubation more than the response to laryngoscopy.

Devavrat Vaishnav et al ${ }^{19}$ (2016) concluded that in patients with ASA Grade I and II, intravenous bolus dose of Esmolol $(2 \mathrm{mg} / \mathrm{kg}$ ) and Fentanyl $(2 \mathrm{mcg} / \mathrm{kg})$ given 3 minutes prior to laryngoscopy and intubation is safe and effective prophylactic method for attenuating haemodynamic response to laryngoscopy and intubation.

Jasleen Kaur et $\mathrm{al}^{20}$ (2013) concluded that butorphanol 20 $\mu \mathrm{g} / \mathrm{kg}$ reduces the induction requirement of propofol comparable to that of fentanyl $2 \mu \mathrm{g} / \mathrm{kg}$ and confers haemodynamic stability at induction and intubation.

Hemangini et $\mathrm{al}^{21}$ (2016) concluded that Butorphanol 20 $\mu \mathrm{g} / \mathrm{kg}$ gives better attenuation of the haemodynamic response, longer duration of postoperative pain relief without producing excessive sedation and with negligible side effects in comparison with fentanyl $1 \mu \mathrm{g} / \mathrm{kg}$ when given intravenously as premedicant for general anaesthesia.

Mean arterial pressure is a derived value and is important for the maintenance of autoregulatory function of the vital organs. Rate pressure product (HR x SBP) is a measure of myocardial oxygen demand. Both Mean Arterial Pressure and Rate Pressure Product increased in both the groups on intubation and the increase was significantly lower in the butorphanol group compared to the fentanyl group.

Both the drugs provided stable haemodynamics throughout the intraoperative period, which was also observed in Philip BK et al study.

In our study, we observed that at the doses at which the study drugs were used both could prevent haemodynamic response to a certain extent. However, butorphanol had a significant advantage over an equipotent dose of fentanyl in the sense that the increase in the stress response was comparatively less and of shorter duration.

\section{Summary}

We observed that there was a significant rise in heart rate, systolic BP, diastolic BP, mean arterial pressure and rate pressure product when compared to preoperative values in both fentanyl and butorphanol group following laryngoscopy and intubation.

We also observed that the haemodynamic response following laryngoscopy and intubation was significantly lower in the butorphanol group than in the fentanyl group.

The increase in the heart rate, diastolic BP, mean arterial pressure and rate pressure product was short-lived in the butorphanol group than the fentanyl group.

\section{CONCLUSION}

With this study we conclude that the administration of intravenous butorphanol prior to induction of anaesthesia helps on better attenuation of the haemodynamic response to laryngoscopy and endotracheal intubation than intravenous fentanyl. Neither of the drugs was associated with any adverse haemodynamic events. Hence, we conclude that butorphanol could be an effective alternative to fentanyl for the attenuation of the haemodynamic stress response to laryngoscopy and endotracheal intubation.

\section{Acknowledgement}

We are very grateful to the Professors and Assistant Professors of the Department of General Surgery.

We are extremely thankful to the Assistant Professors and the Postgraduates of the Department of Anaesthesiology for their help in carrying out this study.

We are thankful to the Institutional Ethical Committee for their guidance and approval for this study. Last but not the least, we thank all our patients for willingly submitting themselves for this study.

We also wish to state that no financial or material support were obtained for this study.

\section{REFERENCES}

1. Bedford RF, Feinstein B. Hospital admission blood pressure: a predictor for hypertension following endotracheal intubation. Anaesth Analg 1980;59(5):36770.

2. Stoelting RK. Blood pressure and heart rate changes during short duration laryngoscopy for tracheal intubation: influence of viscous or intravenous lidocaine. Anaesth Analg 1978;57(2):197-9.

3. Martin DE, Rosenberg H, Aukburg SJ, et al. Low dose fentanyl blunts circulatory response to tracheal intubation. Anaesth Analg 1982;61(8):680-4.

4. Stoelting RK. Attenuation of blood pressure response to laryngoscopy and tracheal intubation with Sodium Nitroprusside. Anaesth Analg 1979;58(2):116-9.

5. Kamra S, Wig J, Sapru RP. Topical nitroglycerine. A safeguard against pressor response to tracheal intubation. Anaesthesia 1986;41(11):1087-91.

6. Mikawa K, Nishina K, Maekawa N, et al. Comparison of nicardipine, diltiazem and verapamil for controlling the cardiovascular responses to tracheal intubation. $\mathrm{Br} \mathrm{J}$ Anaesth 1996;76(2):221-6.

7. Nami K, Takahi K, Tanaka K, et al. Comparison of landiolol and esmolol for attenuation of cardiovascular response and plasma renin activity against tracheal intubation with laryngoscopy. Anaesthesiology 2005;103:433.

8. Karl. Insertion of LMA in place of endotracheal intubation to attenuate the cardiovascular response. IJA 1999;43:305.

9. Kumar. Blocking glossopharyngeal and superior laryngeal nerves to attenuate the cardiovascular response to laryngoscopy and endotracheal intubation. IJA 1993;41:20-5.

10. Stoelting RK, Hiller SC. Handbook of pharmacology and physiology in anesthetic practice. $2^{\text {nd }}$ edn. Philadelphia, PA, USA: Lippincot Williams \& Wilkins 2006:100-1.

11. Miller RD. Basics of anaesthesia. $6^{\text {th }}$ edn. Elsevier Saunders 2011. P. 125. 
12. Prys-Roberts C, Melochie R, Foex P. Studies of anaesthesia in relation to hypertension. I. Cardiovascular responses of treated and untreated patients. $\mathrm{Br} J$ Anaesth 1971;43(2):122-37.

13. King BD, Harris LC, Greifenstein FE, et al. Reflex circulatory responses to direct laryngoscopy and tracheal intubation performed during general anaesthesia. Anaesthesiology 1951;12(5):556-66.

14. Corbett JL, Kerr JH, Prys-Roberts C. Cardiovascular disturbances in severe tetanus due to overactivity of sympathetic nervous system. Anaesthesia 1969;24(2): 198-212.

15. Forbes AM, Dally FG. Acute hypertension during induction of anaesthesia and endotracheal intubation in normotensive men. British Journal of Anaesthesia 1970;42(7):618-24.

16. Philip BK, Scott DA, Freiberger D, et al. Butorphanol compared with fentanyl in general anaesthesia for ambulatory laparoscopy. Can J Anaesth 1991;38(2): 183-6.
17. Arora V, Bajwa SJS, Kaur S. Comparative evaluation of recovery characteristics of fentanyl and butorphanol when used as supplement to propofol anaesthesia. Int J App Basic Med Res 2012;2(2):97-101.

18. Adachi YU, Satomoto $M$, Higuchi $H$, et al. Fentanyl attenuates the hemodynamic response to endotracheal intubation more than the response to laryngoscopy. Anesth Analg 2002;95(1):233-7.

19. Devavrat V, Chaudhari A. Attenuation of cadiovascular responses to laryngoscopy and intubation: a comparative study between IV esmolol hydrochloride and fentanyl citrate. International Journal of Science and Research 2016;5(10):903-38.

20. Kaur J, Srilata M, Padmaja D, et al. Dose sparing of induction dose of propofol by fentanyl and butorphanol: a comparison based on entropy analysis. Saudi Journal of Anaesthesia 2013;7(2):128-33.

21. Hemangini M Patel, Bansari N Kantharia. A study on comparison of intravenous butorphanol with intravenous fentanyl for premedication in general anesthesia. National Journal of Medical Research 2016;6(1):89-91. 